\title{
Measurements of diet quality of employees in the workplace with a focus on mental wellbeing outcomes: a systematic scoping review
}

\author{
E.R. Barton ${ }^{1}$, C. Baldwin ${ }^{1}$, M. Whelan ${ }^{2}$ and R. Gibson ${ }^{1}$ \\ ${ }^{1}$ Department of Nutritional Sciences, King's College London, London, UK and \\ ${ }^{2}$ Work Ready Group, British Dietetic Association, Birmingham, UK
}

Productivity, which includes absenteeism, presenteeism, work loss and disability-adjusted life years, is affected by factors including diet, mental health and lifestyle ${ }^{(1)}$. Poor mental wellbeing is estimated to cost employers $£ 33-42$ billion per year with half of the costs relating to presenteeism ${ }^{(2)}$. A priority for employers is to implement strategies for health promoting behaviour to avoid loss of productivity. Diet quality is the overall pattern of an individual's diet and evidence suggests diet quality can influence mental health conditions such as depression and anxiety ${ }^{(3)}$.

The aim of this study was to conduct a scoping review to describe how diet quality is measured in the workplace, and secondly to investigate how mental wellbeing outcomes were measured when associated with diet quality in the workplace.

A systematic scoping review was conducted following the PRISMA extension for scoping reviews (PRISMA-ScR). MEDLINE, EMBASE and CINAHL databases were searched using pre-defined search terms. Titles and abstracts of records were screened and a full-text review for final inclusion. Data was extracted and a data-charting form completed. A narrative synthesis was conducted covering how diet and diet quality is measured in the workplace, and associated workplace and health outcomes. Studies with a focus on mental wellbeing and study characteristics were included in sub- group thematic analysis.

For inclusion there were 46 studies with three of these assessing mental wellbeing outcomes including depression, job strain, stress and sleep. There was a lack of intervention studies $(n=4)$ with one randomised controlled trial. There were 32 different diet intake measurement tools. Food frequency questionnaires were the most common $(n=31)$ of which there 18 different types. Of the 24 different diet quality indices identified across the studies, Dietary Approaches to Stop Hypertension was the most frequently used $(\mathrm{n}=9)$. There were fourteen occupation groups with nurses being the most common $(n=11)$, and 11 types of workplaces investigated in included studies most frequently at universities $(n=6)$. There was variation in outcomes assessed with 76 in total. Of these, one study investigated business outcomes and three examined mental wellbeing outcomes. Of the mental wellbeing outcomes assessed, diet quality measured by DASH scores (OR 1.66) and the Japanese Food Guide Spinning Top ( $p=0.003)$ was associated with job strain but no association with stress, sleep or depression was found.

This review highlights the variability and lack of consistency of diet intake measures, diet quality indices and outcomes measured in existing research of employees in the workplace. In addition, a lack of randomised controlled trials highlights the need for intervention studies in the workplace with standardisation of methods and a focus on mental wellbeing and business outcomes through well- conducted randomised controlled trials.

\section{References}

1. Goetzel R, Stacey R, MS Ozminkowski, et al. (2004) J Occup Environ Med 46, 398-412.

2. Deloitte (2017) [Available at: https://www2.deloitte.com/uk/en/pages/public-sector/articles/mental-health-employers-review.html].

3. Jacka F, Mykletun A, Berk M et al. (2011) Psychosom Med 73, 483-490. 